\title{
ARTICLE
}

\section{Hybrid Graphene Nematic Liquid Crystal Light Scattering Device}

Received ooth January 2012 Accepted ooth January 2012

DOI: $10.1039 /$ xoxxooooox

www.rsc.org/

\author{
M. M. Qasim ${ }^{a *}$, A. A. Khan ${ }^{\text {a }}$ A. Kostanyan ${ }^{\text {a }}$, P. R. Kidambi ${ }^{\mathrm{b}}$, A. Cabrero- \\ Vilatela $^{\mathrm{b}}$, P. Braeuninger-Weimer ${ }^{\mathrm{b}}$, D. J. Gardiner ${ }^{\mathrm{a}}$, S. Hofmann ${ }^{\mathrm{b}}$ and T. D. \\ Wilkinson $^{\mathrm{a}}$
}

\begin{abstract}
A hybrid graphene nematic liquid crystal (LC) light scattering device is presented. This device exploits the inherent poly-crystallinity of chemical vapour deposited (CVD) graphene films to induce directional anchoring and formation of LC multi-domains. This thereby enables efficient light scattering without the need for crossed polarisers or separate alignment layers/additives. The hybrid LC device exhibits switching thresholds at very low electric fields

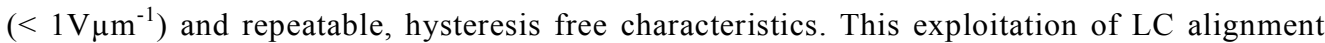
effects on CVD graphene films enables a new generation of highly efficient nematic LC scattering displays as well as many other possible applications.
\end{abstract}

\section{Introduction}

Liquid crystal (LC) interactions and alignment properties at substrate interfaces is a crucial aspect of display technologies and many other LC based applications. It is also one of the most poorly understood areas of display design and is usually derived empirically. Modern LC displays make use of a thin $(\sim 10 \mathrm{~nm})$ rubbed polymer alignment layer on top of a transparent conductive electrode such as indium tin oxide (ITO) ${ }^{1}$ to align the LC molecules. This ITO layer is also normally designed and optimised for performance using empirical techniques. Alternative transparent conductor materials (TCMs) are currently being explored to create new functionalities and form factors. Two dimensional materials such as graphene are of great interest due to their flexibility, chemical inertness combined with their broad optical transparency and unusual electrical properties ${ }^{2,3}$. While

\footnotetext{
a. Centre of Molecular Materials for Photonics and Electronics, Department of Engineering, University of Cambridge, 9 J.J. Thomson Avenue, Cambridge, CB3 OFA, UK.

b. Department of Engineering, University of Cambridge, Cambridge, CB3 OFA, UK.

*. Corresponding author, email: qmm20@cam.ac.uk
}

Footnotes relating to the title and/or authors should appear here. Electronic Supplementary Information (ESI) available: [details of any supplementary information available should be included here]. See DOI: $10.1039 / x 0 x \times 00000 x$ exfoliated graphene flake based LC devices have been explored, they are ultimately limited by the size of individual flakes and the electrical contact made to their nearest neighbours ${ }^{4}$.

Continuous graphene films synthesised using chemical vapour deposition (CVD) are a stronger candidate for advanced TCMs as they can be grown over large areas and transferred easily onto glass substrates allowing many of the processing and manufacturing challenges to be addressed ${ }^{5,6}$.

Earlier research has shown interesting molecular alignment of nematic and smectic LCs with highly oriented pyrolytic graphite (HOPG) $)^{7,8}$. Nematic LCs have also been used to characterise the poly-crystalline basal domain structure and defects of as-grown graphene CVD films ${ }^{9}$. The directional anchoring and formation of LC multidomains on the CVD graphene has been partly explained by $\pi$-stacking interactions, but this LC alignment effect is not well understood or explored ${ }^{9,10,11}$. Further, graphene based LC devices has also been utilized for phase modulation of terahertz electromagnetic fields ${ }^{12}$.

Here, we show that the LC alignment effects of CVD grown graphene films can be exploited to generate additional functionality when used as a TCM in display 
applications, creating a new generation of nematic LC based scattering displays. Current light scattering devices require a complicated or multistep fabrication process ${ }^{13}$ and typically use polymer dispersed LCs to achieve scattering domains in a polymer matrix ${ }^{14,15,16,17}$. Other variants include mixtures of negative and positive dielectric anisotropy $(\Delta \varepsilon)$ nematic $\operatorname{LCs}^{18,19}$ or ionically doped smectic (SmA) LC scattering materials that use electro-hydrodynamic instabilities (EHDI) to achieve scattering 20,21 . These SmA devices, albeit exhibiting strong optical scattering, suffer from lifetime and stability issues due to electro chemical degradation of the ionic medium ${ }^{20,22}$. We demonstrate a simple hybrid graphene nematic LC light scattering device that utilises the as-grown poly-crystallinity of the CVD graphene electrode to allow light scattering without the need for polarisers or separate alignment layers/additives, capable of switching with very a low electric field $\left(<1{\left.\mathrm{~V} \mu \mathrm{m}^{-1}\right)}^{-1}\right.$ threshold and repeatable, hysteresis free characteristics.

\section{Experimental \\ Synthesis of graphene films}

Details of synthesis and extensive characterization of the CVD graphene can be found in our prior work ${ }^{23,24}$.

The as-grown mono-layer graphene film is transferred from the $\mathrm{Cu}$ foil to a pre-cleaned glass substrate by using a polymer support layer of polystyrene (PS, Mw 35k, 2\% $\mathrm{w} / \mathrm{w}$ in toluene) and an acid $\left(\mathrm{FeCl}_{3} \mathrm{aq}, 0.5 \mathrm{M}\right)$ to etch the $\mathrm{Cu}$. This was followed by a wash in a warm ethyl acetate bath to dissolve the supporting polymer layer. The graphene film is characterised by Raman spectroscopy, scanning electron microscopy (SEM) and polarising optical microscopy (Supplementary data, Figure S1).

\section{Device fabrication}

Figure 1(a) shows the LC cell design based on glass substrates. Four sets of cells were fabricated using different TCMs; Gr (graphene)-Gr, Gr-ITO (Figure 1b), ITO-ITO with additional polymer alignment layers on both electrodes (anti-parallel rubbed polyimide,) and ITO-ITO without any alignment layers. Commercial display ITO coated glass (Instrument Glasses Inc. UK) was used. For $\mathrm{Gr}$ electrodes a metallic thin film contact (Ni, $\sim 50 \mathrm{~nm}$ ) was deposited on one edge of the glass before the graphene transfer to form an electrical contact point. The electrodes were bonded together using Norland $68 \mathrm{UV}$ glue that contained $10 \mu \mathrm{m}$ spacer beads to set a $10 \mu \mathrm{m}$ cell gap.
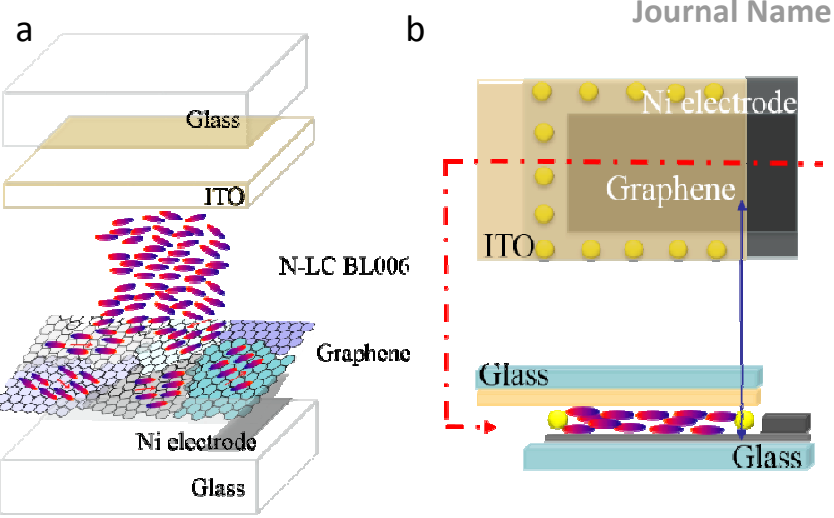

Figure 1 A schematic of Gr-ITO $10 \mu \mathrm{m}$ cell filled with nematic liquid crystal, (a) overall device operation (b) cell structure of the Gr-ITO cell.

A commercial nematic liquid crystal mixture (BL006, Merck Co., Germany, clearing point of $113{ }^{\circ} \mathrm{C}, \Delta \mathrm{n}=0.28$ and $\Delta \varepsilon=17.3$ ) was used to fill the cells by capillary action. All four set of cells were systematically examined using a polarising optical microscope (POM), Olympus BX-60, and optical micrographs were recorded for each of the devices under different electric fields and with a crossed polariser arrangement (Supplementary data, Table S1).

\section{Results and Discussion}

Initially these devices were examined using a polarising optical microscope (Olympus BX-60) and optical micrographs were collected under crossed polarisers. An

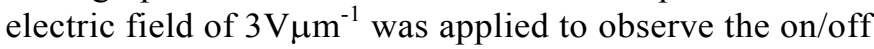
state of each device (Supplementary data Table 1, depicts all the micrographs). The polarising optical micrographs show some very unusual results. Test cells with a graphene conductive layer exhibited a LC multi-domain texture (protuberant irregular bright spots with black background), which were noticeable visible under crossed polarisers with and without applied electric field.

Representative polarising optical micrographs (POM) images of the $10 \mu \mathrm{m}$ thick Gr-ITO switching cell under crossed polarisers with (b-h) and without (a) applied electric field are shown in Figure 2. Micrographs were recorded at low magnification $10 \mathrm{x}$, and the depth of the focus was kept above the surface of graphene sheet to view a maximum area of switching device. The cells with graphene conductive layer showed LC multi-domain texture (irregular bright areas with a black background), which were visible under crossed polarisers, in particular Figure 2(b-d) displayed a layer of liquid crystal as Schlieren, thread like, texture. The presence of this multidomain switching and the absence of a complete dark state (homeotropic texture) in the graphene test cells are indicative of a scattering behaviour in the nematic LC devices.

However, when characterizing ITO-ITO test cells, LC multi-domains were only observed in the cells without 
any additive alignment layers. Under applied field both ITO-ITO test cells (with and without alignment layer) exhibited a homeotropic texture, i.e. the texture appeared as a dark state in the optical micrograph between crossed polarisers, indicating full switching of the nematic LC molecules (Supplementary data, Table S1).

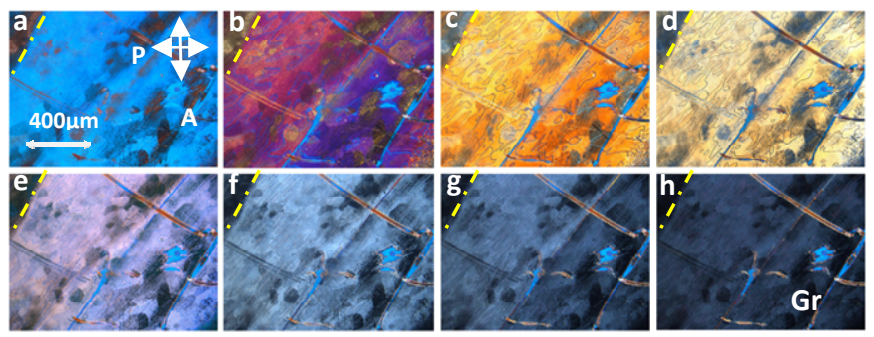

Figure 2 POM images of the $10 \mu \mathrm{m}$ thick Gr-ITO switching cell under crossed polarisers. LC homeotropic alignment under applied electric field a) No field applied, (b) $0.6 \mathrm{~V} / \mu \mathrm{m}$, c) $0.75 \mathrm{~V} / \mu \mathrm{m}$, d) $0.9 \mathrm{~V} / \mu \mathrm{m}$, e) $1.5 \mathrm{~V} / \mu \mathrm{m}$, f) $1.8 \mathrm{~V} / \mu \mathrm{m}, \mathrm{g}) 2.1$ $\mathrm{V} / \mu \mathrm{m}$ ) and $\mathrm{h}$ ) $3 \mathrm{~V} / \mu \mathrm{m}$ field (yellow dotted line is the edge of the graphene sheet (Gr) and scale bars: $400 \mu \mathrm{m})$.

In order to find the magnitude of this scattering behaviour, polariser-free transmission measurements were collected using a collimated light emitting diode (LED) excitation setup (532 nm, variable power) shown in Figure 3. Light from the LED was incident on the cells and the transmitted light was focused onto a silicon photo-detector (Thorlabs PDA10A). A function generator (TG1304, Thurlby Thandar) was connected to a variablegain voltage amplifier (built in-house) to apply electric fields across the test cells, and transmission data was recorded with a digitizing oscilloscope (HP54503 A, Hewlett Packard) and logged on computer.

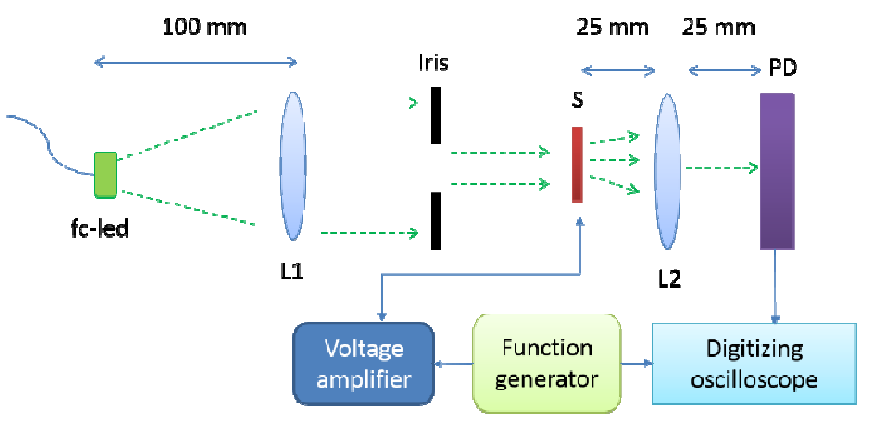

Figure 3 Experimental setup used to measure light scattering characteristics of the test cells. L1: $100 \mathrm{~mm}$ focal length lens, L2: $25 \mathrm{~mm}$ focal length lens, S: mounted sample cell, LED: $532 \mathrm{~nm}$ cantered, fibre coupled led light source, the iris aperture was set to $4 \mathrm{~mm}$, and a collimated beam of light was incident on the sample.

The unpolarized light transmission was measured as a function of the electric field applied to each cell, and the effective director reorientation as a function of scattering was compared across the cells. Figure 4 summarises the light scattering characteristics for each cell configuration.
The ITO-ITO cells with alignment layers have demonstrated only small change in transmission by increasing electric field (Figure 4). Whereas, ITO-ITO cells without alignment layers has shown around 10\% change in the transmittance. However, a considerable change in transmission was observed in the case of the cells with graphene electrodes, $40 \%$ and $25 \%$ in the GrITO and Gr-Gr cell structures respectively (for Gr-ITO device, transmission lowest $35 \%$ to highest $75 \%$, Gr-Gr device transmission lowest $45 \%$ to highest $65 \%$ ). This is attributed to the onset of scattering from the LC domains formed by LC alignment on graphene (Figure 2). The ITO-ITO cell with alignment layers showed the highest transmission due to additional polymer additive which gave uniform texture across the device (Supplementary data, Table 1). While in case of ITO-ITO cells without the alignment layers, it is possible, the liquid crystal molecules are randomly aligned due to the absence of any alignment layers. So under applied electric field liquid crystals are switching from one random to another random domain orientation without any significant effect on the scattering of incident unpolarized light.

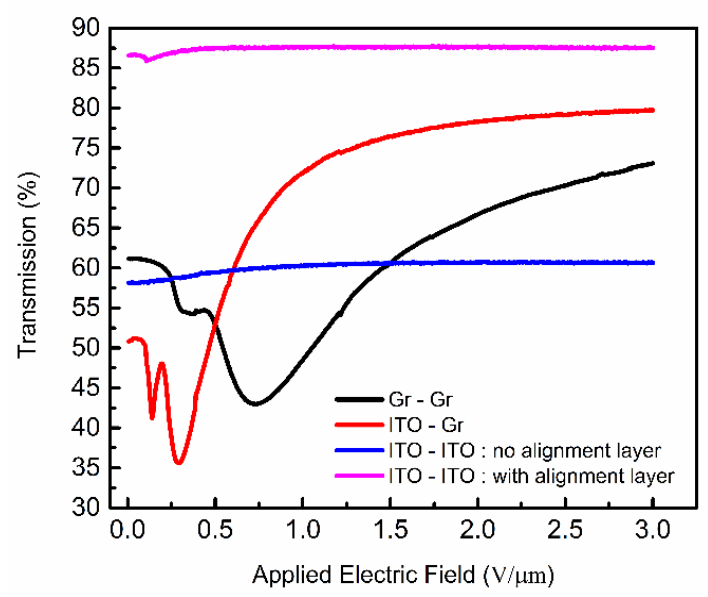

Figure 4 Transmission vs. applied electric field traces for the various cell configurations. $10 \mu \mathrm{m}$ LC BL006 filled cells.

On the other hand, in Gr-cells the molecules tend to align according to the polycrystalline graphene structure. While under an applied electric field these aligned molec sles reorient with respect to the applied field and the graphene domains. Hence we observe significant chang $?$ in transmittance.

Importantly, for both graphene devices (Gr-Gr, Gr-ITO), the tiansmission spectra show an initial fall in transmission upon the application of an electric field followed by a subsequent increase in the magnitude of transmission. This indicates an initial alignment of the individual domains with the applied electric field, yielding a partial homeotropic texture within the domains 
before a bulk transition to the scattering state in the material (Supplementary data, Figure S2).

The observed behaviour for the Gr-Gr and Gr-ITO electrode cells (highest transmission $\sim 25 \%$ and $\sim 40 \%$ respectively shown in Figure 4) shows a significant improvement over the results seen with the ITO-ITO cells(without any additives), and opens up the possibility of simple high contrast displays utilising only the nematic LC material as the scattering medium. Furthermore, we emphasize that all devices have shown switching thresholds at very low electric fields $\left(<1 \mathrm{~V} \mu \mathrm{m}^{-}\right.$ $\left.{ }^{1}\right)$.

As it has been reported before ${ }^{25}$, the observed scattering behaviour can be rationalised via a simple analysis of the device using LC continuum theory. The conventional out-of-plane electric field induced switching of a nematic LC is governed by the magnitude of $\mathrm{K}_{11}$ (splay deformation). The Frederick's transition for a $10 \mu \mathrm{m}$ BL006 filled cell where $\mathrm{K}_{11}=17.9 \times 10^{-12}$, is equal to an applied electric field magnitude of $0.12{\mathrm{~V} \mu \mathrm{m}^{-1}}^{-}$Eq. 1 shows this switching magnitude relation ${ }^{1}$.

$$
\mathrm{E}_{\mathrm{th}}=\pi / \mathrm{d} \sqrt{ }\left(\mathrm{K}_{11} /\left(\varepsilon_{\mathrm{o}} \Delta \chi_{\mathrm{e}}\right)\right.
$$$$
\text { eq. } 1
$$

where $\mathrm{d}$ is the thickness of the LC film and $\Delta \chi \mathrm{e}$ is the anisotropy of the electric susceptibility of the LC (BL006 in this case). This is an ideal case where a single LC domain switches from a homogeneous to homeotropic alignment or vice versa.

The interaction between the LC layer and the polycrystalline lattice of the graphene surface is very complex and similar to degenerate planar alignment ${ }^{26}$. This frustrated planar interaction leads to the formation of random LC domains of different sizes and different director orientations at the interface of the graphene lattice. Also, there is a possibility that different graphene domains with different orientations may distort the electric field profile inside the cell causing varying effects on the different LCs orientations. On increasing the field magnitude, the initial increase in scattering is due to an increase in refractive index disorder that is a result of micron sized LC domains that have different switching thresholds, and hence a mixture of switched, un-switched and partially switched domains could possibly form, leading to randomly switched LC layers that cause visible light to scatter in different orientations. At higher field levels, the splay deformation overcomes this random switching and all of the LC layers align with the external field, leading to increased transmission of the light.

To further test the hypothesis that the graphene layer leads to a disordered poly-domain LC texture, dielectric measurements were performed on these four test devices (dielectric measurements of all four test cells are in the supplementary data, Figure S5). Figure 5 shows the comparison between ITO-ITO with alignment layers and Gr-ITO cells. The sandwich cell capacitances were compared before and after LC filling, followed by the application of the E-field to quantify the change in effective relative permittivity of the bulk LC switching medium. It can be seen from Figure 5, that the relative permittivity measured is higher in the ITO-ITO cell (17.3), as compared to the ITO-Gr cell (15.9). BL006, is a positive dielectric LC, hence higher permittivity correspond to better alignment of the molecular long axis with the applied electric field. Thus, the measured relative permittivities are indicative of incomplete field induced homeotropic alignment, and correspond to the LC micro-domain texture that leads to the observed light scattering behaviour.

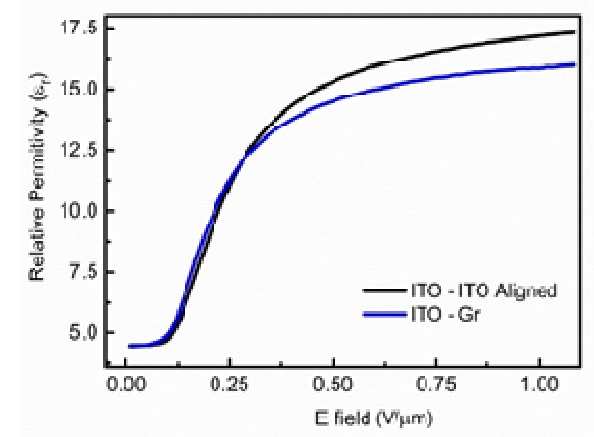

Figure 5 Relative permittivity vs applied E-field permittivity in BL006 LC filled ITO-ITO and ITO-Gr cells. E-field induced LC reorientation shows different alignment layer induced bulk response.

The crystallographic nature of the CVD graphene together with the level of impurities and defects significantly impacts the LC domain formation which in turn impacts the optical properties of the device. The microstructure of the graphene film thereby intimately relates to the CVD conditions and can be engineered via control over nucleation density and domain shape evolution during the graphene CVD process ${ }^{23,24}$. The average basal domain size for the mono-layer graphene films used here was 5-10 $\mu \mathrm{m}$, as characterised by SEM, Raman and optical microscopy (Supplementary data, Figure S1). Light can get scattered at defects such as domain boundaries as well as other graphene imperfections such as defects, wrinkles, cracks, stress points and impurities (in particular polymer residue) introduced during the growth and transfer processes.

In addition to the scattering characteristics reported above, we also compared the response times of the different test cells, which are summarized in Table 1.Once again, there is a significant disparity among the cells, and the electrodes played an important role on each device's response times. As expected, the rise times were highest for the Gr-Gr cells, and lowest for the ITO-ITO cells without alignment layers. This is a very important observation, as it shows that the level of multi-domain interaction between the LC layers in the graphene cells is much higher compared to the ITO-ITO cells. 
Correspondingly, the trend is reverse for the fall times, and we see that the fall time is lowest for the Gr-Gr cell, and highest for the ITO-ITO cell without alignment layers. This is further confirmation of the strength of interaction of these LC domains with the surface. Overall switching times were measured to be lowest for the Gr-ITO cell, as it represents a trade-off between the amount of anchoring, leading to fast rise times and small fall times, the latter close to those seen for $\mathrm{Gr}-\mathrm{Gr}$ electrodes.

\begin{tabular}{|l|c|c|c|}
\hline Devices & $\begin{array}{c}\text { Rise } \\
\text { Time } \\
(\mathrm{ms})\end{array}$ & $\begin{array}{c}\text { Fall Time } \\
(\mathrm{ms})\end{array}$ & $\begin{array}{c}\text { Total } \\
\text { switching } \\
\text { time }(\mathrm{ms})\end{array}$ \\
\hline Gr-Gr & 5.1 & 6.1 & 11.1 \\
\hline Gr-ITO & 0.9 & 6.8 & 7.7 \\
\hline ITO-ITO & 0.4 & 41.5 & 41.9 \\
\hline ITO-ITO AL & 0.5 & 31.0 & 31.4 \\
\hline $\begin{array}{c}\text { Devices with } \\
\text { Dichoric Black } \\
\text { dye (S428) }\end{array}$ & $\begin{array}{c}\text { Rise } \\
\text { Time } \\
(\mathrm{ms})\end{array}$ & $\begin{array}{c}\text { Fall Time } \\
(\mathrm{ms})\end{array}$ & $\begin{array}{c}\text { Total } \\
\text { switching } \\
\text { time }(\mathrm{ms})\end{array}$ \\
\hline Gr-Gr & 37.5 & 4.5 & 42.0 \\
\hline Gr-ITO & 1.9 & 8.9 & 10.8 \\
\hline ITO-ITO & 0.5 & 72.5 & 73.0 \\
\hline ITO-ITO AL & 0.7 & 58.0 & 58.7 \\
\hline
\end{tabular}

Table 1 Response times of the test cells, rise time refers to the time interval between $10 \%$ and $90 \%$ of the increase in transmission on the application of a $3{\mathrm{~V} \mu \mathrm{m}^{-1}}^{-1}, 1 \mathrm{KHz}$ electric field and fall time (relaxation time) on electric field removal.

To observe the repeatability of the scattering state within the Gr based devices repetitive test examinations were operated on a Gr-Gr electrode cell (Figure 6b). Five transmission tests for the same Gr-Gr electrode cell filled with nematic LC show minimal variation in the scattering characteristics. Another aspect we investigated was the variation of the scattering characteristics with the frequency of the applied electric field 25. We tested the Gr-Gr cell across a frequency range from $100 \mathrm{~Hz}$ to $10 \mathrm{kHz}$ and did not find any frequency dependency on its scattering behaviour (Figure 6a).

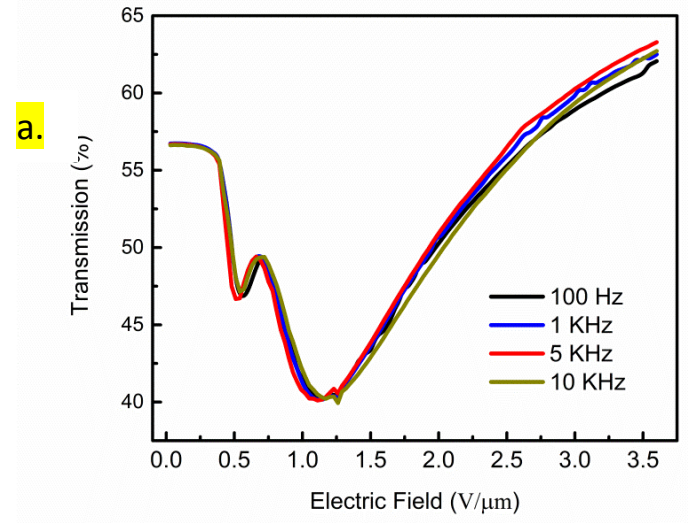

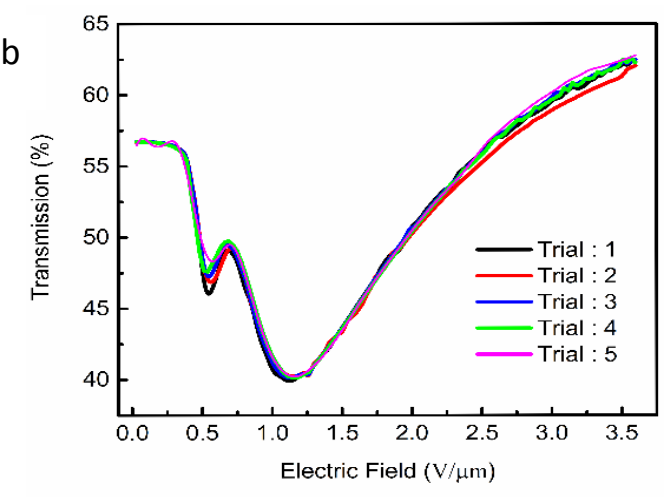

Figure 6 Transmission curves of a Gr-Gr cell filled with $10 \mu \mathrm{m}$ BL006 nematic LC, (a) the effect of applied electric field and frequency on the scattering characteristic of the test cell (b) repeated switching runs.

Finally, we demonstrate a working device with an optical scattering behaviour under an ordinary white light source at room temperature using a Gr-ITO test cell (Supplementary data, Figure S3, S4). Literature suggests that to enhance and to promote coloured/white opaque initial state of the LC scattering device, high molecular weight materials can be utilized to tune the LC scattering device to a transparent state under an applied electric field. In our study, dichroic dye (S428 ${ }^{28}$,Mitsui, Japan) has been added to observe black-to-transparent state, whereas reactive mesogen RM257, Merck, ${ }^{14,18,28}$ has been used to observe opaque white-to-transparent state.

Both doped Gr-ITO devices exhibited an enhanced scattering effect with only small amounts of these dopants (4\% w/w S428 and $1 \%$ w/w of RM257) presented in Figure 7. The transmission curves show a different trend as compare to Figure 4 because, firstly, the dichroic dye is absorbing, so it decreases the overall amplitude of light transmission (even in the switched state). Secondly, addition of a dye changes the composition of the switching LC medium and so the interaction that is leading to the observed scattering effect this often leads to the shape of the curves change slightly in response.

Further to add this doping amount was much lesser than that seen with previous non-graphene TCM literature. 27,28 . The removal of the scattering state into the clear state with an applied electric field was sufficiently strong in both cases to allow the clear view of printed text placed behind each cell as shown in Figure 8 (b \& d). 


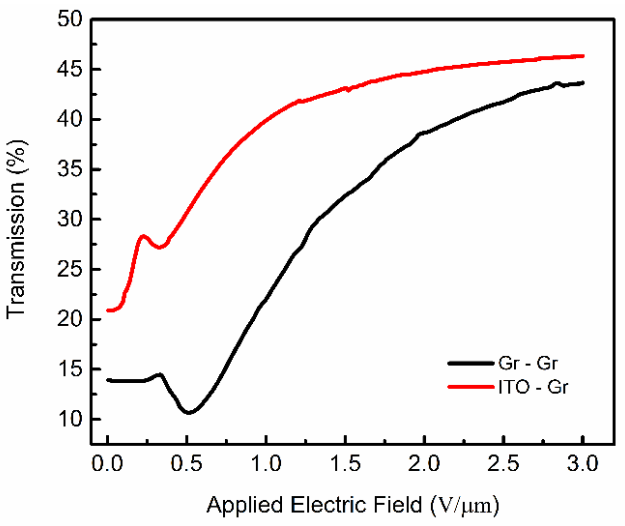

Figure 7 Transmission vs. applied electric field traces for $10 \mu \mathrm{m}$ Gr-Gr and ITO-Gr devices filled with BL006 N$\mathrm{LC}+4 \%$ black dye.

A Cambridge University monogram was placed underneath the Gr-ITO test cell. Images were collected with and without electric field (Figure $8 \mathrm{a}-\mathrm{b}$, device is filled with S428 doped nematic LC mixture whereas Figure $8 \mathrm{c}-\mathrm{d}$ shows scattering behaviour with RM257 in nematic LC mixture fill in Gr-ITO device).
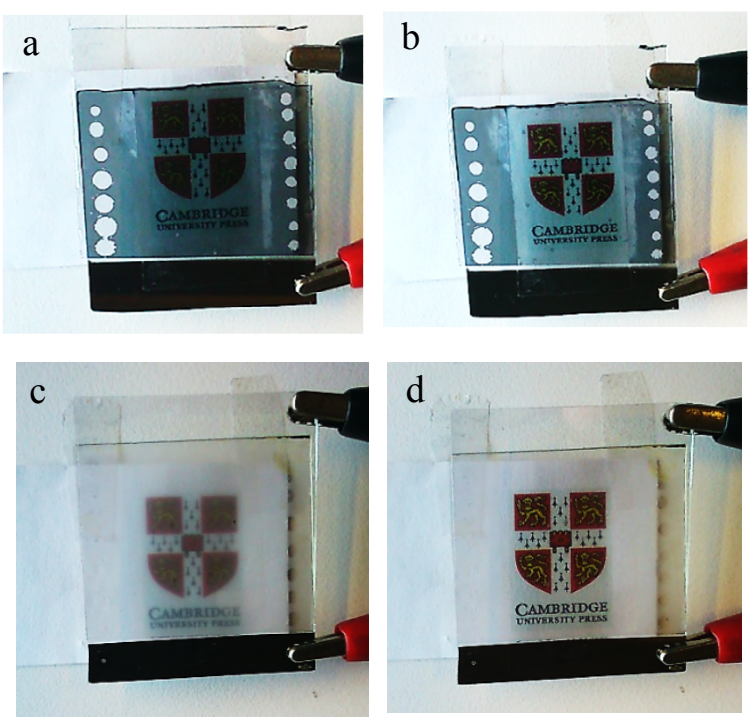

Figure 8 Test cell Gr-ITO electrodes with $10 \mu \mathrm{m}$ spacer beads, filled with a mixture of (a,b) BL006 nematic LC with 4\% black dye S428, and (c,d) BL006 with 1\% RM257. (a,c) No electric field applied and (b,d) under applied electric field, $3 \mathrm{~V} \mu \mathrm{m}^{-1}, 1 \mathrm{kHz}$.

\section{Conclusions}

We have shown how LC alignment effects on graphene CVD films can be exploited as an additional functionality for TCM layers in LC devices. In this work we compared
ITO and graphene based LC cell configurations and show that the as-grown poly-crystallinity of the CVD graphene films induces directional anchoring and formation of LC multi-domains which generates efficient light scattering without the need for crossed polarisers or separate alignment layers or special LC materials. Graphene electrodes exhibited higher rise times (from scattering to clear transition), but lower fall (relaxation) times, when compared to ITO reference electrodes. Hence the lowest total response times can be achieved with a hybrid GrITO cell design, which also shows the highest scattering contrasts. The hybrid LC devices exhibit switching

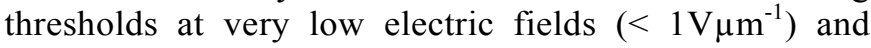
repeatable, hysteresis free characteristics. Our data opens up new possibilities for future devices and a new generation of highly efficient low cost nematic LC scattering displays, combining the advantages of the different TCM materials.

\section{Acknowledgements}

Funding from the EPSRC (Grant No. EP/K016636/1, GRAPHTED) is acknowledged. P.R.K. acknowledges funding from Cambridge Commonwealth Trust (CCT) and the Lindemann Trust Fellowship. A.A.K would like to thank the Higher Education of Pakistan (HEC) and the CCT for financial support. A.C.V. acknowledges funding from the Cambridge Conacyt Scholarship and the Roberto Rocca Fellowship. A.K. would like to thank the Luys Educational Foundation and Hovnanian Foundation for scholarships.

\section{References}

P. J. Collings and M. Hird, Introduction to Liquid Crystals: Chemistry and Physics. CRC Press, 1997.

K. Ellmer, "Past achievements and future challenges in the development of optically transparent electrodes," Nat. Photonics, vol. 6, no. 12, pp. 809-817, Nov. 2012.

S. Bae, S. J. Kim, D. Shin, J.-H. Ahn, and B. H. Hong, "Towards industrial applications of graphene electrodes," Phys. Scr., vol. T146, no. T146, p. 014024, Jan. 2012.

P. Blake, P. D. Brimicombe, R. R. Nair, T. J. Booth, D. Jiang, F. Schedin, L. a. Ponomarenko, S. V. Morozov, H. F. Gleeson, E. W. Hill, A. K. Geim, and K. S. Novoselov, "Graphene-based liquid crystal device," Nano Lett., vol. 8, no. 6, pp. 1704-1708, 2008.

P. R. Kidambi, C. Weijtens, J. Robertson, S. Hofmann, and J. Meyer, "Multifunctional oxides for integrated manufacturing of efficient graphene electrodes for organic electronics," vol. 063304, pp. 1-6, 2015. 
[6] A. Zurutuza and C. Marinelli, "Challenges and opportunities in graphene commercialization.," Nat. Nanotechnol., vol. 9, no. 10, pp. 730-734, Oct. 2014.

[7] J. S. Foster and J. E. Frommer, "Imaging of liquid crystals using a tunnelling microscope," Nature, vol. 333, no. 6173, pp. 542-545, Jun. 1988.

[8] M. Shigeno, W. Mizutani, M. Suginoya, M. Ohmi, K. Kajimura, and M. Ono, "Observation of Liquid Crystals on Graphite by Scanning Tunneling Microscopy," Jpn. J. Appl. Phys., vol. 29, no. Part 2, No. 1, pp. L119-L122, Jan. 1990.

[9] D. W. Kim, Y. H. Kim, H. S. Jeong, and H.-T. Jung, "Direct visualization of large-area graphene domains and boundaries by optical birefringency.," Nat. Nanotechnol., vol. 7, no. 1, pp. 29-34, Jan. 2012.

[10] D. P. Singh, S. K. Gupta, T. Vimal, and R. Manohar, "Dielectric, electro-optical, and photoluminescence characteristics of ferroelectric liquid crystals on a graphenecoated indium tin oxide substrate," Phys. Rev. E, vol. 90, no. 2, p. 022501, Aug. 2014.

[11] Y. U. Jung, K.-W. Park, S.-T. Hur, S.-W. Choi, and S. J. Kang, "High-transmittance liquid-crystal displays using graphene conducting layers," Liq. Cryst., vol. 41, no. 1, pp. 101-105, 2013.

[12] T. Sasaki, K. Noda, N. Kawatsuki, and H. Ono, "Universal polarization terahertz phase controllers using randomly aligned liquid crystal cells with graphene electrodes.," Opt. Lett., vol. 40, no. 7, pp. 1544-7, Apr. 2015.

[13] J. Chen, W. Cranton, and M. Fihn, Eds., Handbook of Visual Display Technology. Berlin, Heidelberg: Springer Berlin Heidelberg, 2014.

[14] Y. H. Lin, H. Ren, and S. T. Wu, "High contrast polymerdispersed liquid crystal in a 90 twisted cell," Appl. Phys. Lett., vol. 84, no. 20, pp. 4083-4085, 2004.

[15] C. Lampert, "Smart switchable glazing for solar energy and daylight control," Sol. Energy Mater. Sol. Cells, vol. 52, no. 3-4, pp. 207-221, 1998.

[16] A. Fuh and O. Caporaletti, "Polymer dispersed nematic liquid crystal films: The density ratio and polymer's curing rate effects," J. Appl. Phys., vol. 66, no. 11, pp. 5278-5284, 1989.

[17] Y.-D. Chen, A. Y.-G. Fuh, and K.-T. Cheng, "Particular thermally induced phase separation of liquid crystal and poly(N-vinyl carbazole) films and its application," Opt. Express, vol. 20, no. 15, p. 16777, 2012.

[18] J. W. Doane, N. a. Vaz, B. G. Wu, and S. Žumer, "Field controlled light scattering from nematic microdroplets," Appl. Phys. Lett., vol. 48, no. 4, pp. 269-271, 1986.
[19] H. Pleiner, R. Stannarius, and W. Zimmermann,

"Electrically Driven Instabilities in Smectic Liquid Crystal Films," vol. 295, pp. 285-324, 1998.

[20] M. V. Mitrokhin, L. Johnsen, R. Fagerberg, C. Kristiansen, F. J. Farrand, K. H. Holm, R. Palm, K. Netland, and V. L. Aristov, "Prevention of Smectic-A LC Mixtures Electrolytic Degradation," Mol. Cryst. Liq. Cryst., vol. 411, no. 1, pp. 255-263, 2004.

[21] D. J. Gardiner and H. J. Coles, "Enhancing lifetime in a bistable smectic A liquid crystal device," J. Phys. D. Appl. Phys., vol. 40, no. 4, pp. 977-981, 2007.

[22] A. A. Khan, S. M. Morris, D. J. Gardiner, M. M. Qasim, T. D. Wilkinson, and H. J. Coles, "Improving the stability of organosiloxane smectic A liquid crystal random lasers using redox dopants," Opt. Mater. (Amst)., vol. 42, pp. 441-448, Apr. 2015

[23] P. R. Kidambi, B. C. Bayer, R. Blume, Z. J. Wang, C. Baehtz, R. S. Weatherup, M. G. Willinger, R. Schloegl, and S. Hofmann, "Observing graphene grow: Catalyst-graphene interactions during scalable graphene growth on polycrystalline copper," Nano Lett., vol. 13, no. 10, pp. 4769-4778, 2013.

P. R. Kidambi, C. Ducati, B. Dlubak, D. Gardiner, R. S. Weatherup, M. B. Martin, P. Seneor, H. Coles, and S. Hofmann, "The parameter space of graphene chemical vapor deposition on polycrystalline Cu," J. Phys. Chem. C, 2012

[25] A. A. Khan, G. D. M. R. Dabera, H. Butt, M. M. Qasim, G. A. J. Amaratunga, S. R. P. Silva, and T. D. Wilkinson, "Tunable scattering from liquid crystal devices using carbon nanotubes network electrodes," Nanoscale, 2015.

[26] I. Dierking, F. Gießelmann, and P. Zugenmaier, "TGB A state in a homologous series of diarylethane $\alpha$-chloroester ferroelectric liquid crystals," Liq. Cryst., vol. 17, no. 1, pp. 17-22, Jul. 1994.

[27] D. J. Gardiner and H. J. Coles, "Organosiloxane liquid crystals for fast-switching bistable scattering devices," $J$. Phys. D. Appl. Phys., vol. 39, no. 23, pp. 4948-4955, 2006.

[28] D. J. Gardiner, S. M. Morris, and H. J. Coles, "Highefficiency multistable switchable glazing using smectic A liquid crystals," Sol. Energy Mater. Sol. Cells, vol. 93, no. 3, pp. 301-306, 2009. 


\section{Supplementary Information:}

\section{Hybrid Graphene Nematic Liquid Crystal Light Scattering Device}

M. M. Qasim*, A. A. Khan, A. Kostanyan, P. R. Kidambi, A. Cabrero-Vilatela, D. J. Gardiner, P. Braeuninger-Weimer, S. Hofmann and T. D. Wilkinson.

Department of Engineering, University of Cambridge, 9 J.J. Thomson Avenue, Cambridge, CB3 0FA, UK.

*Corresponding author: qmm20@.cam.ac.uk

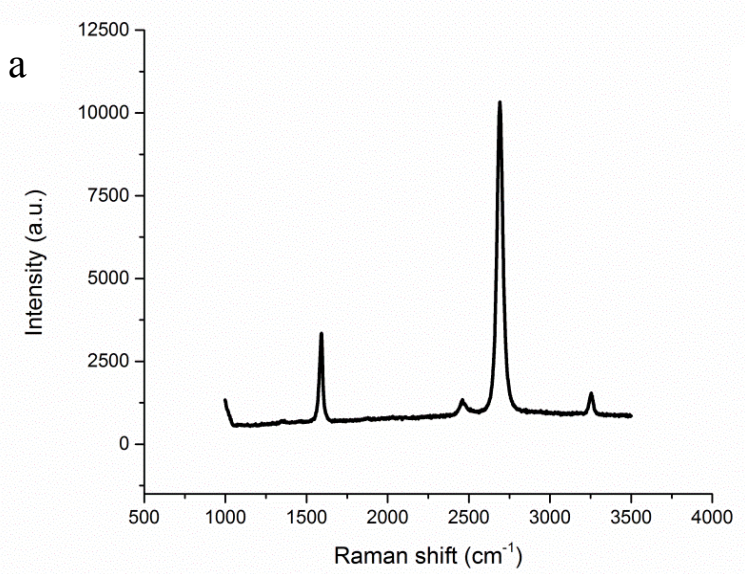

$\mathrm{b}$

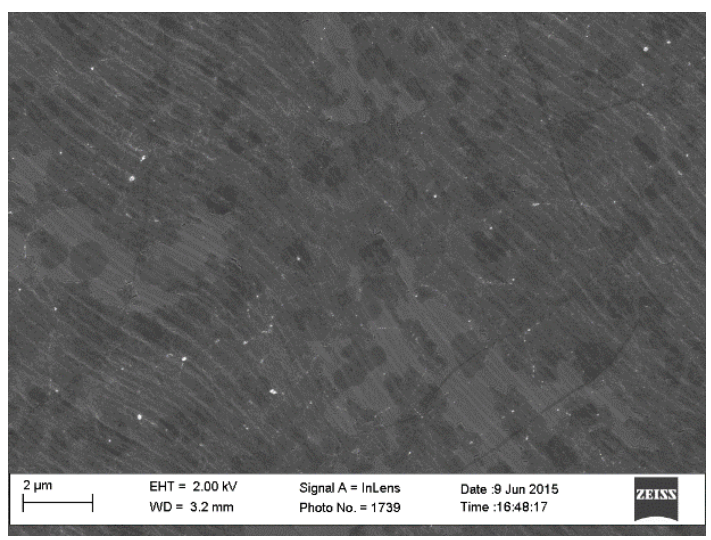

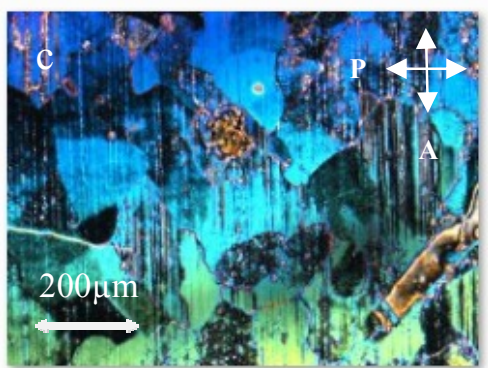

1

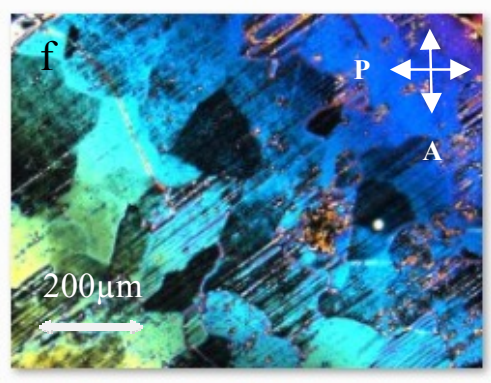

4

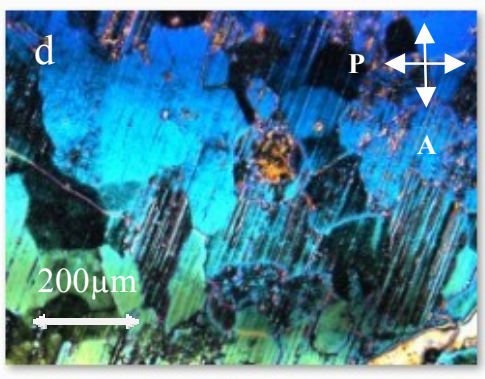

2

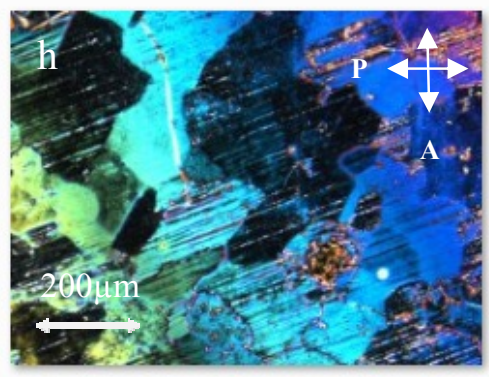

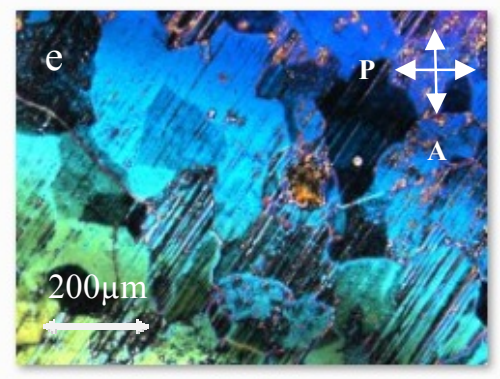

3

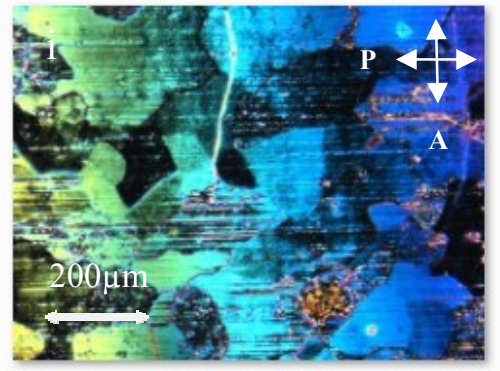

Figure S1 Characterisation of graphene layer after the transfer a) Raman spectrum and b) SEM image of graphene transferred onto the silicon substrate. Optical micrographs of Gr-glass substrate with spin-coated nematic liquid crystal texture on rotation c) $0^{\circ}$ degrees and i) $90^{\circ}$ degrees under crossed polariser arrangement. 


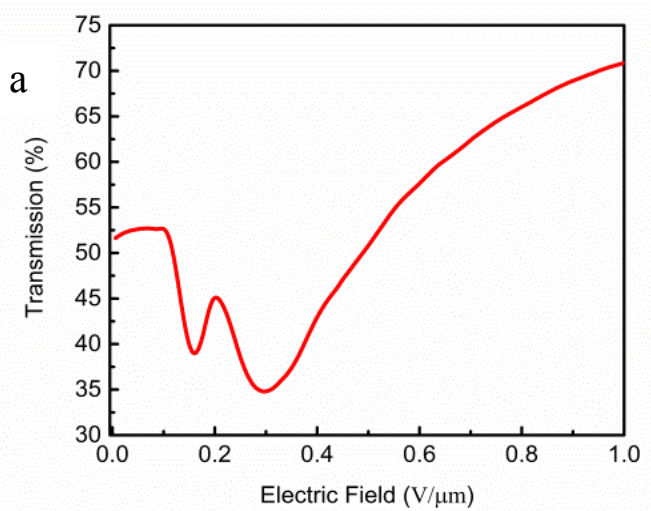

Figure S2: The transmission characteristics oscillations earlier presented in figure 3.

(a) Transmission vs. applied electric field for $10 \mu \mathrm{m}$ Gr-ITO device filled with nematic LC, (b-d) corresponding POM micrographs. From no field applied (b) and $0.4 \mathrm{~V} / \mu \mathrm{m}(\mathrm{c})$ there is a very dramatic change in the observed texture, which corresponds to the detected dips in transmission. This further leads to $0.45 \mathrm{~V} / \mu \mathrm{m}(\mathrm{d})$ where the texture is recovered showing rise in transmission.
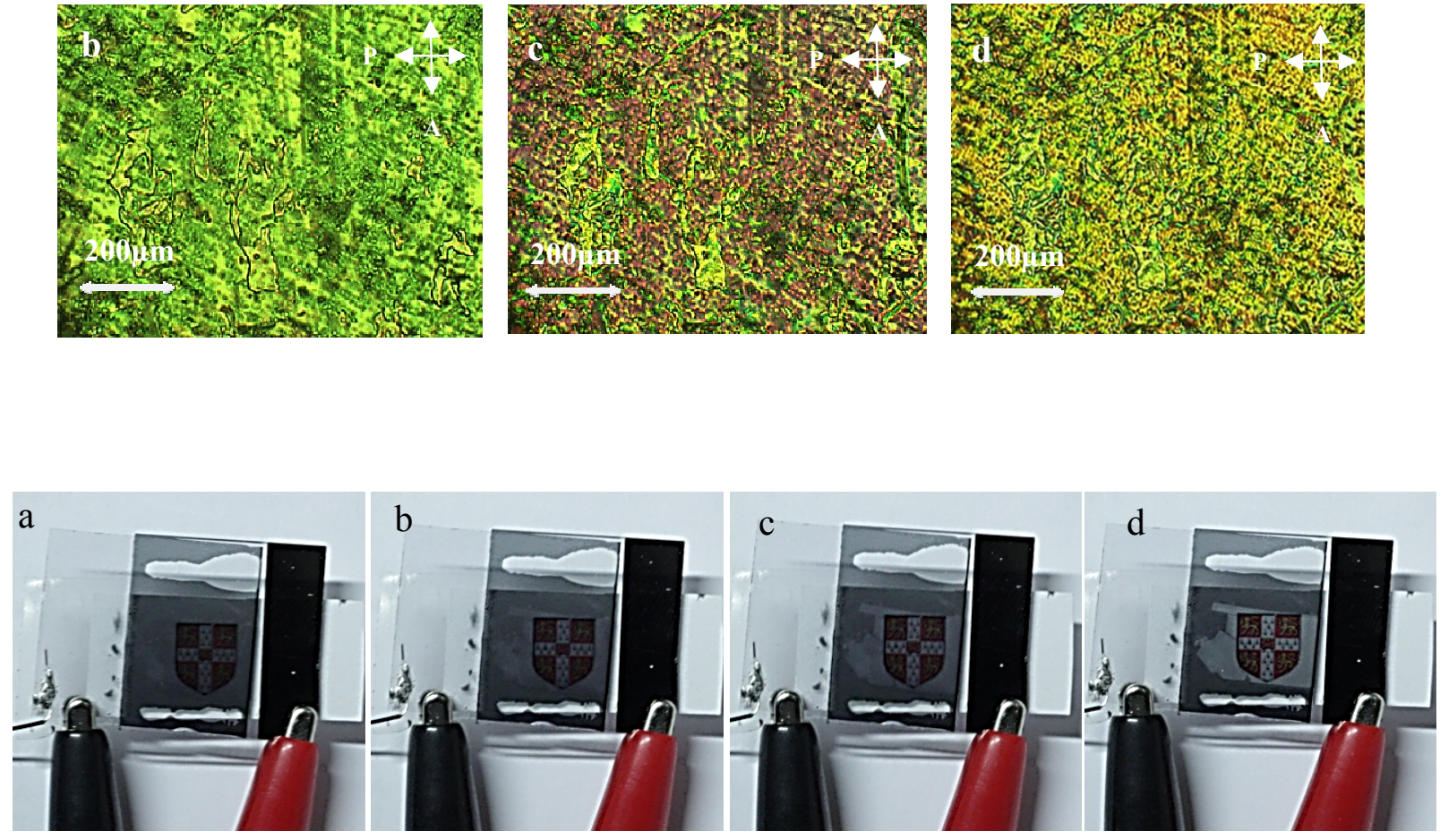

Figure S3 Demonstration of the scattering to clear transition with Gr-ITO switching cell filled with dye doped BL006 nematic LC mixture. Figures (a) to (d) show a gradual change in transmission under applied electric field 0-3 V/ $\mu \mathrm{m}, 1$ $\mathrm{kHz}$.

a

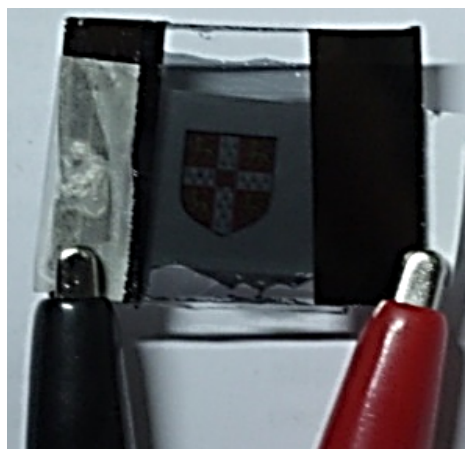

$\mathrm{b}$

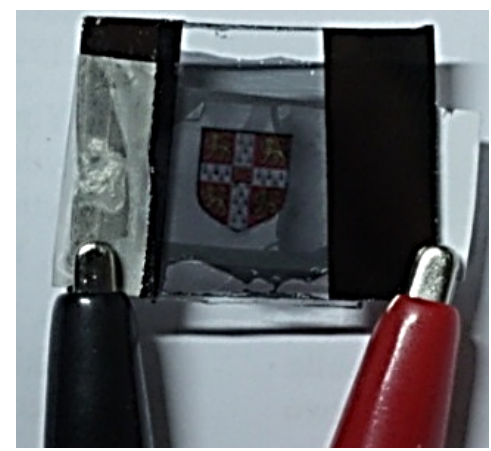

Figure S4 Demonstration of scattering in ambient room lighting for dye doped BL006 nematic LC mixture filled in Gr-Gr cell, (a) off-state, no applied electric field and (b) on-state, $3 \mathrm{~V} / \mu \mathrm{m} 1 \mathrm{kHz}$. 

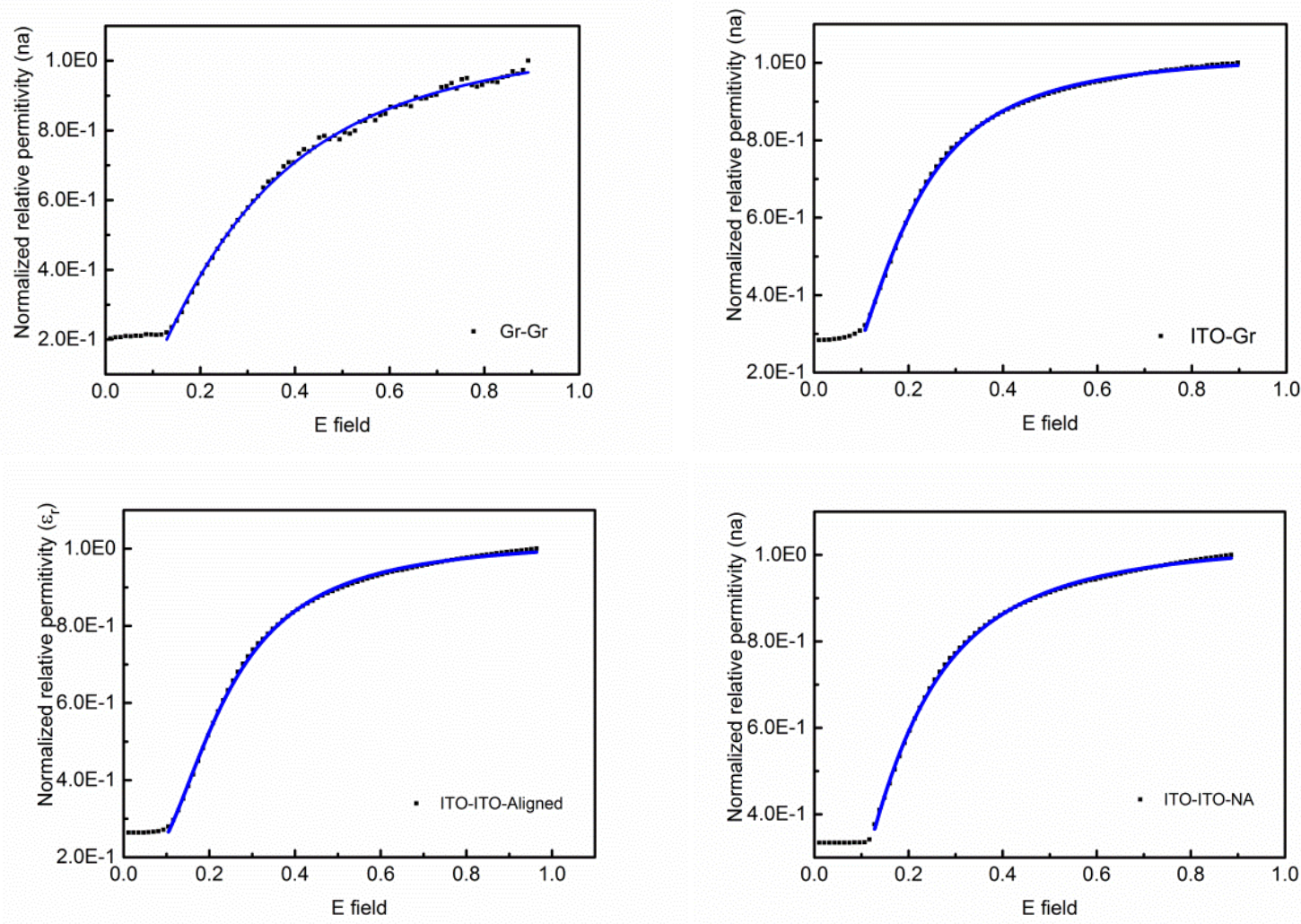

Figure S5 The normalized dielectric permittivity plots for four types of substrate geometries sandwiched in $10 \mu \mathrm{m}$ thickness devices filled with nematic liquid crystal a) Gr-Gr, b)Gr-ITO, c) ITO-ITO with and d)ITO-ITO without alignment layer. 
No field applied crossed polarizers
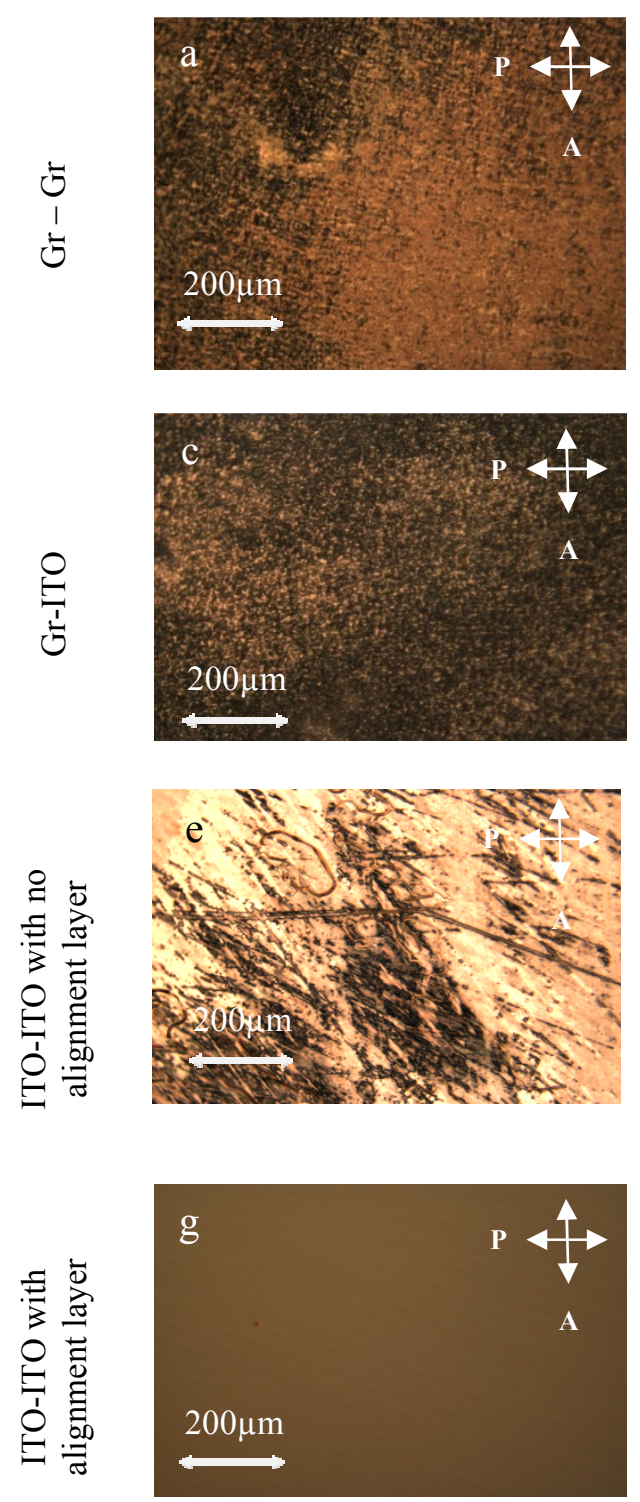

Field applied crossed polarizers
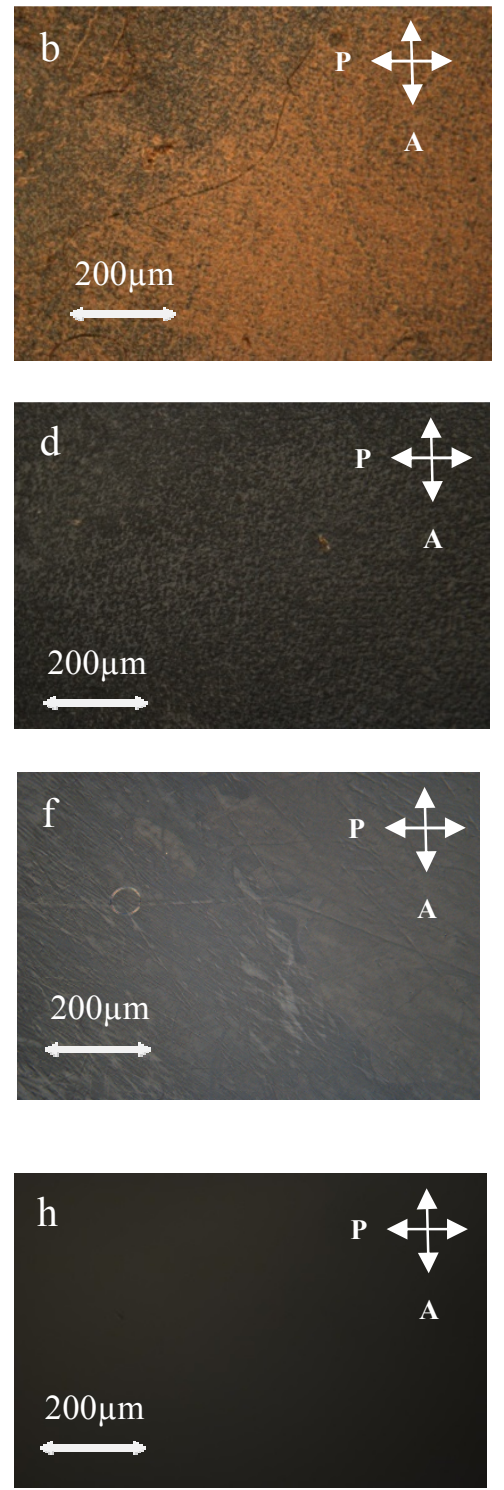

Table S1 Optical micrographs of test cells using different TCM with $10 \mu \mathrm{m}$ spacer beads filled with nematic BL006 LC. For texture characterization images were taken with crossed polarized light and with and without applied electric field $(3 \mathrm{~V} / \mu \mathrm{m}, 1$ $\mathrm{kHz}$ and scale bars: $200 \mu \mathrm{m}$, magnification $20 \mathrm{x}$ ). 